\title{
Ciliary adenylyl cyclases control the Hedgehog pathway
}

\author{
Laura Vuolo, Antonio Herrera, Blanca Torroba, Anghara Menendez and Sebastian Pons*
}

\begin{abstract}
Protein kinase $A(P K A)$ accumulates at the base of the cilium where it negatively regulates the Hedgehog $(\mathrm{Hh})$ pathway. Although PKA activity is essentially controlled by the cAMP produced by adenylyl cyclases, the influence of these enzymes on the Hh pathway remains unclear. Here, we show that adenylyl cyclase 5 and adenylyl cyclase 6 (AC5 and AC6, also known as ADCY5 and ADCY6, respectively) are the two isoforms most strongly expressed in cerebellar granular neuron precursors (CGNPs). We found that overexpression of AC5 and AC6 represses, whereas their knockdown activates, the $\mathrm{Hh}$ pathway in CGNPs and in the embryonic neural tube. Indeed, AC5 and AC6 concentrate in the primary cilium, and mutation of a previously undescribed cilium-targeting motif in AC5 suppresses its ciliary location, as well as its capacity to inhibit $\mathrm{Hh}$ signalling. Stimulatory and inhibitory $\mathrm{G} \alpha$ proteins, which are engaged by the G-protein-coupled receptors (GPCRs), control AC5 and AC6 activity and regulate the Hh pathway in CGNPs and in the neural tube. Therefore, we propose that the activity of different ciliary GPCRs converges on AC5 and AC6 to control PKA activity and, hence, the $\mathrm{Hh}$ pathway.
\end{abstract}

KEY WORDS: Adenylyl cyclase, Cerebellar granular neuron precursor, Hedgehog pathway, Primary cilium

\section{INTRODUCTION}

Sonic hedgehog (Shh) belongs to the Hedgehog (Hh) family of secreted signalling proteins. Shh activity is crucial during the development of the nervous system to control the proliferation and the dorso-ventral patterning of the neural tube (Dessaud et al., 2008; Le Dréau and Martí, 2012), and for the clonal expansion of cerebellar granular cell precursors (CGNPs; Dahmane and Ruiz i Altaba, 1999; Wechsler-Reya and Scott, 1999). In vertebrates, most of the Hh signalling pathway components are located at or in the proximity of the primary cilium, and the integrity of this organelle is an absolute requirement for correct Hh signalling (Briscoe and Thérond, 2013). Indeed, in the absence of $\mathrm{Hh}$, the 12-pass transmembrane protein that receives Hh ligands, Patched 1 (Ptch1), is concentrated in and around the primary cilium (Rohatgi et al., 2007). However, upon Hh binding, Ptch1 is excluded from the cilium and the G-proteincoupled receptor (GPCR) Smoothened (Smo) concentrates there (Corbit et al., 2005). Gli proteins are the only known transcriptional effectors of the Hh pathway and the activity of the pathway is fully dependent on the balance between activator and repressor forms of Gli2 and Gli3. In addition, protein kinase A (PKA), the main repressor of the Hh pathway, also accumulates at the base of the cilium (Barzi et al., 2010), where activation by cAMP induces

Instituto de Biología Molecular de Barcelona (CSIC), Parc Científic de Barcelona, Baldiri Reixac 10-12, Barcelona 08028, Spain.

*Author for correspondence (spfbmc@ibmb.csic.es)

Received 2 April 2015; Accepted 15 June 2015 repression driven by Gli2 and Gli3 (Wang et al., 2000; Tempe et al., 2006; Tuson et al., 2011). Although the activity of PKA is basically regulated by CAMP, it remains unclear to what extent the adenylyl cyclases, which produce cAMP from ATP, influence the $\mathrm{Hh}$ pathway.

Adenylyl cyclases are currently grouped into six classes that can be distinguished on the basis of their sequence. All adenylyl cyclases found in metazoans belong to the class III adenylyl cyclases, and in mammals, class III adenylyl cyclases can be further divided into membrane-bound adenylyl cyclases (type 1 to type 9, AC1-AC9, also known as ADCY1-ADCY9) and soluble adenylyl cyclases (sACs; Linder, 2006). The nine membrane-bound adenylyl cyclases are controlled by a complex set of factors, including heterotrimeric G-proteins. Stimulatory G-proteins $\left(\mathrm{G} \alpha_{\mathrm{s}}\right)$ activate all nine adenylyl cyclase isoforms to some extent, whereas inhibitory G-proteins $\left(\mathrm{G}_{\mathrm{i}}\right)$ only inhibit AC1, AC5 and AC6 (Linder, 2006; Sadana and Dessauer, 2009). Smo is a fully competent activator of Go $\alpha_{i}$ proteins (Shen et al., 2013) and in Drosophila, Smo acts as a canonical GPCR that signals through $\mathrm{G \alpha}_{\mathrm{i}}$ in order to regulate the Hh pathway (Ogden et al., 2008). Moreover, in rat CGNPs, simultaneous knockdown of $\mathrm{G \alpha}_{\mathrm{i}} 2$ and $\mathrm{G \alpha}_{\mathrm{i}} 3$ (also known as GNAI2 and GNAI3, respectively) dramatically hinders the proliferation of these cells induced by Shh (Barzi et al., 2011). By contrast, GPR161, an orphan GPCR that engages $\mathrm{G} \alpha_{\mathrm{s}}$, exerts negative effects on the Hh pathway (Mukhopadhyay et al., 2013). Furthermore, targeted knockout of $G N A S$, the gene that encodes $\mathrm{G} \alpha_{\mathrm{s}}$, causes medulloblastoma-like tumours in mouse cerebellum (He et al., 2014).

In this work, we show that AC5 and AC6 are the two adenylyl cyclase isoforms most strongly expressed in two well established Shh-target tissues: CGNPs and the embryonic neural tube. We also show that overexpression of AC5 and AC6 represses the Hh pathway, whereas their knockdown results in consistent activation of the Hh pathway, both in CGNPs and the neural tube. In addition, we show that AC5 and AC6 concentrate in the primary cilium and that the mutations of a previously undescribed motif in AC5 suppress its targeting to the cilium and its capacity to inhibit the $\mathrm{Hh}$ pathway. Finally, we demonstrate that, as in CGNPs, the $\mathrm{Hh}$ pathway is positively and negatively regulated by $\mathrm{G} \alpha_{\mathrm{i}}$ and $\mathrm{G} \alpha_{\mathrm{s}}$ in the neural tube, respectively. Therefore, we propose that the activity of different ciliary GPCRs converges on AC5 and AC6 to control PKA activity and, hence, the Hh pathway.

\section{RESULTS}

Different adenylyl cyclase isoforms are expressed in the developing external germinative layer of the cerebellum The proliferation of CGNPs in the external germinative layer (EGL) of the cerebellum (Fig. 1A) depends entirely on Shh activity and consequently, this tissue has proved to be an excellent model to study the regulation of the Hh pathway. Conveniently, CGNPs can be easily purified and cultured (Rios et al., 2004). When we studied the expression of the nine membrane-bound adenylyl cyclase isoforms in purified mouse CGNPs by quantitative real-time PCR 


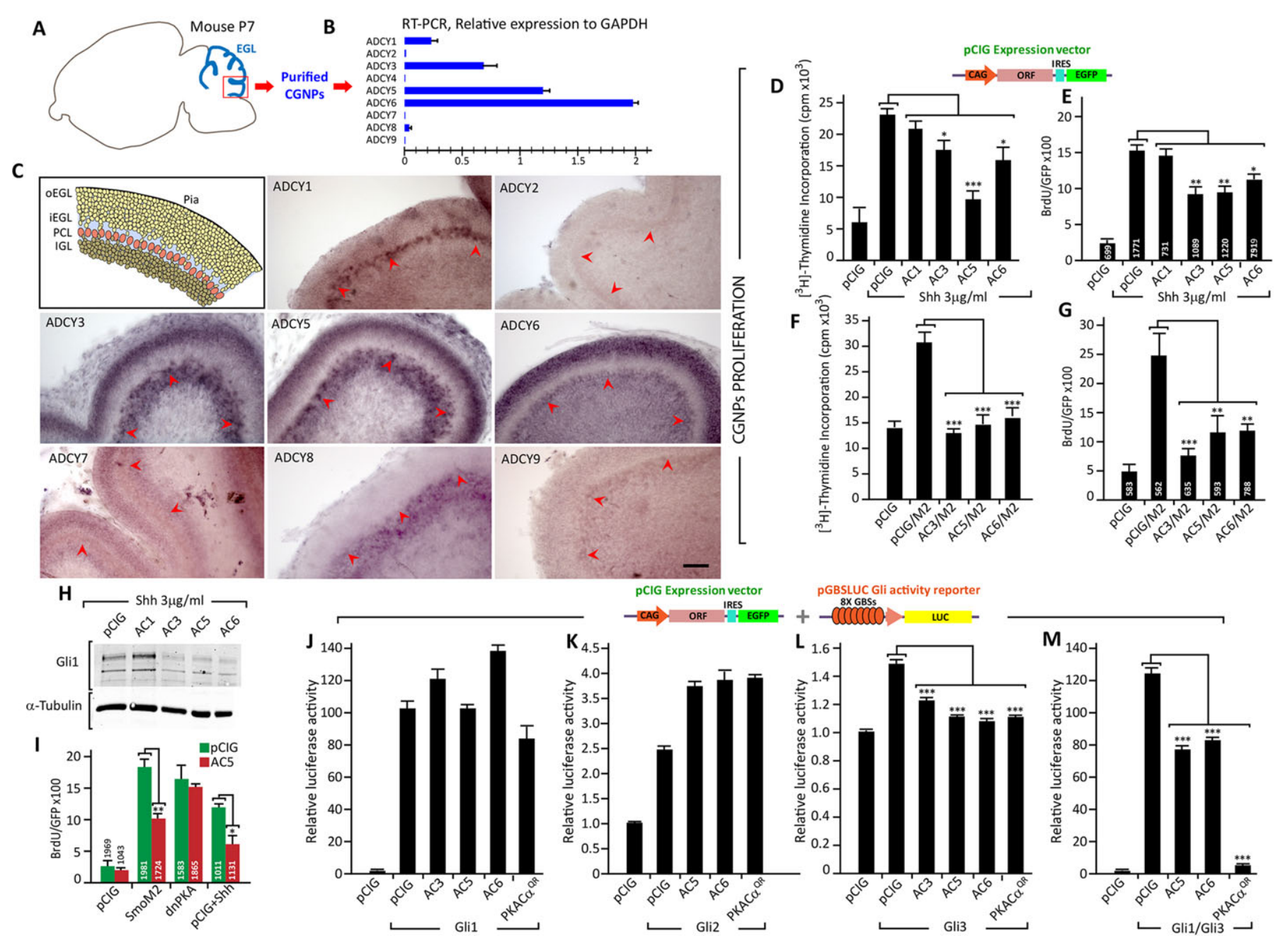

Fig. 1. Adenylyl cyclase isoforms expressed by CGNPs inhibit the Hh pathway. $(A, B)$ Expression of the different membrane-bound adenylyl cyclases in CGNPs purified from the P7 mouse cerebellum as evaluated by q-PCR. (C) Expression of the same adenylyl cyclases (except AC4) studied by in situ hybridization. The red arrowheads indicate the position of the Purkinje cell layer and the relative position of each cell layer in the P7 cerebellum is shown (oEGL, iEGL, outer and inner external germinative layer; PCL, Purkinje cell layer; IGL, internal granular layer). (D,E) Proliferation of CGNPs studied by $\left.{ }^{3} \mathrm{H}\right] \mathrm{thymidine}$ or BrdU incorporation (a pulse for the last $4 \mathrm{~h}$ in culture) in cultures transfected with AC1, AC3, AC5 or AC6 and grown for $24 \mathrm{~h}$ in the presence of $3 \mu \mathrm{g} / \mathrm{ml}$ of Shh. A diagram of the expression vector is shown above the graphs. $(F, G)$ Experiment similar to that described in $D, E$ but in this case CGNP proliferation was induced by transfecting a constitute-active form of Smo (SmoM2). For BrdU incorporation, the bars represent the percentage of the transfected population (GFP) that incorporated BrdU. (H) Gli1 in western blots of CGNP cultures transfected for $24 \mathrm{~h}$ with different adenylyl cyclase isoforms. (I) Effect of the expression of AC5 on CGNPs in culture where proliferation was induced by the expression of SmoM2, dominant-negative (dn)PKA or the addition of Shh (3 $\mu \mathrm{g} / \mathrm{ml})$. (J-M) GBS-

dependent luciferase activity was studied in CGNP cultures transfected for $24 \mathrm{~h}$ with Gli1, Gli2, Gli3 or combined Gli1 and Gli3 and the different adenylyl cyclase isoforms. A constitutively active form of PKA (PKAC $\alpha^{\mathrm{QR}}$ ) was used as a positive control. A diagram of the expression vectors is shown above the graphs. All the bar graphs in this figure show the mean \pm s.d. of at least three independent experiments. The total number of cells counted for each data point in panels $E, G$ and $I$ is indicated inside each bar. ${ }^{*} P<0.05,{ }^{* *} P<0.01,{ }^{* *} P<0.001$ (one-way ANOVA followed by the Tukey's test). Scale bar: $50 \mu \mathrm{m}$.

(q-PCR), we found that AC5 and AC6 were the most strongly expressed isoforms, followed by $\mathrm{AC} 3$ and $\mathrm{AC} 1$, whereas $\mathrm{AC} 2, \mathrm{AC} 4$, AC7, AC8 and AC9 were only weakly expressed (Fig. 1B). In fact, $\mathrm{AC} 3, \mathrm{AC} 5$ and AC6 were also the most strongly expressed isoforms in the cerebellar EGL when studied by in situ hybridization (ISH). It is worth noting that AC6 was the only adenylyl cyclase isoform that in cerebellum was almost exclusively expressed in the EGL.

\section{The proliferation of CGNPs induced by Shh is inhibited by AC3, AC5 and AC6 but not by AC1}

Forskolin is an allosteric activator of adenylyl cyclases and dibutyril-cAMP (DBA) is a cAMP analogue, both of which have been reported to inhibit the Hh pathway (Dahmane and Ruiz i Altaba, 1999; Wechsler-Reya and Scott, 1999; Rios et al., 2004).
Given that the effect of adenylyl cyclase expression on the Hh pathway had not been assessed previously, we measured the proliferation of CGNPs expressing AC1, AC3, AC5 or AC6 that was induced by Shh. Notably, we found that Shh-induced proliferation was significantly reduced in CGNPs ectopically expressing AC3, AC5 and AC6 but not in those expressing AC1, as reflected by both $\left[{ }^{3} \mathrm{H}\right]$ thymidine (Fig. 1D) and BrdU (Fig. 1E) incorporation. Indeed, similar results were obtained when the Hh pathway was activated by transfecting CGNPs with a constitutively active form of Smo, SmoM2 (Fig. 1F,G). In addition, the endogenous expression of Gli1 (a direct target of the pathway) was significantly diminished in cultured CGNPs grown in the presence of Shh when they expressed AC3, AC5 and AC6 but not AC1 (Fig. 1H). In fact, the effect of AC5 expression could be reverted by a dominant-negative form of PKA 
but not by SmoM2, confirming that the activity of adenylyl cyclases lies between Smo and PKA in the Hh pathway (Fig. 1I).

\section{Suppression of the Shh pathway by adenylyl cyclases is mediated by $\mathrm{Gli3}$ in CGNPs}

To further understand how adenylyl cyclases regulate the $\mathrm{Hh}$ pathway we used the GBS-Luc construct, a Luciferase reporter driven by a Gli-binding site (GBS). None of the adenylyl cyclases, nor an active form of PKA $\alpha$ catalytic subunit $\left(\mathrm{PKAC} \alpha^{\mathrm{QR}}\right)$, used as control, reduced GBS-Luc activity when the pathway was activated by Gli1 or Gli2 transfection (Fig. 1J,K). Rather, and perhaps paradoxically, AC5, AC6 and PKAC $\alpha^{\mathrm{QR}}$ increased Gli2-stimulated GBS-Luc activity (Fig. 1K). However, although Gli3 only mildly activated luciferase expression when compared to Gli1 or Gli2, this activation was significantly dampened by AC3, AC5, AC6 and PKAC $\alpha^{\mathrm{QR}}$ (Fig. 1L). Moreover, if Gli3 was co-expressed with Gli1 the suppression of GBS-Luc activity induced by adenylyl cyclases, and especially by $\mathrm{PKAC} \alpha^{\mathrm{QR}}$, was significantly stronger (Fig. 1M). In addition we confirmed that the formation of the repressor form of Gli3 was reduced by SmoM2 and increased by AC5 or PKAC $\alpha^{\mathrm{QR}}$ (supplementary material Fig. S1A). These data are in agreement with the elevated capacity of Gli3 to drive Hh pathway inhibition compared to Gli1 or Gli2 (Pan et al., 2006). Thus, together these results indicate that $\mathrm{AC} 3, \mathrm{AC} 5$ and $\mathrm{AC} 6$ but not $\mathrm{AC} 1$ can antagonize Hh signalling in CGNPs, possibly by modulating Gli activity.

\section{Adenylyl cyclases must be localized to cilia to regulate the Hh pathway}

Although cAMP is a fully diffusible metabolite, its activity is largely constricted by phosphodiesterases. Thus, because PKA regulates the Hh pathway from the base of the cilium (Barzi et al., 2010; Tuson et al., 2011), a ciliary or peri-ciliary distribution of the adenylyl cyclases regulating PKA would also be expected. AC3 has previously been shown to accumulate at the primary cilium in CGNPs (Barzi et al., 2010); however, whether it regulates the Hh pathway was not known. To address this issue, we transfected CGNPs with HA-tagged constructs encoding the different adenylyl cyclase isoforms endogenously expressed in these cells (AC1, AC3, AC5 and AC6), and we found AC3, AC5 and $\mathrm{AC} 6$ but not $\mathrm{AC} 1$ at the cilium (Fig. 2A). Additionally, using
A

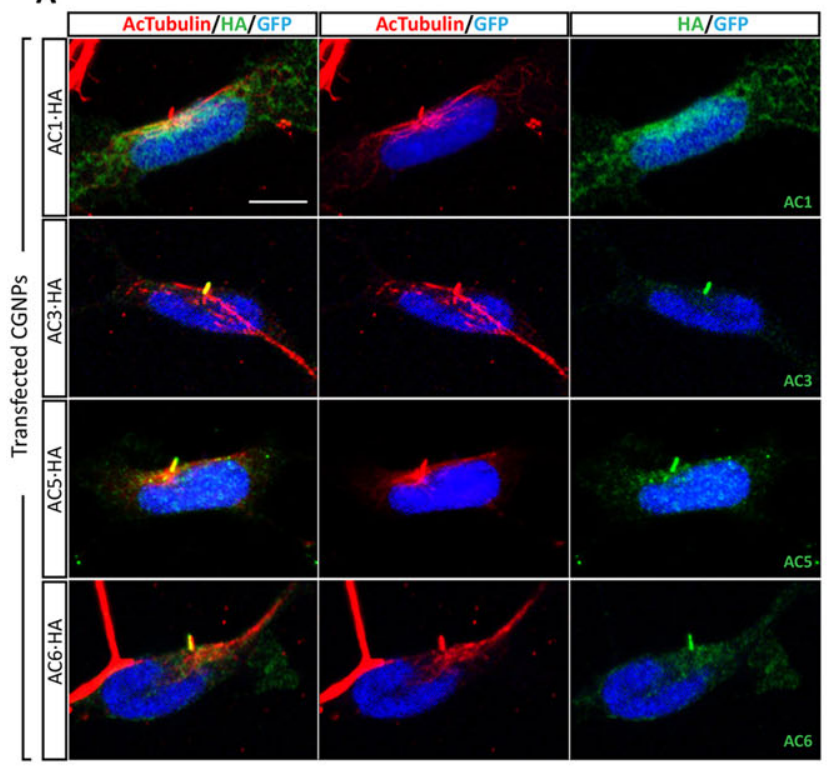

B

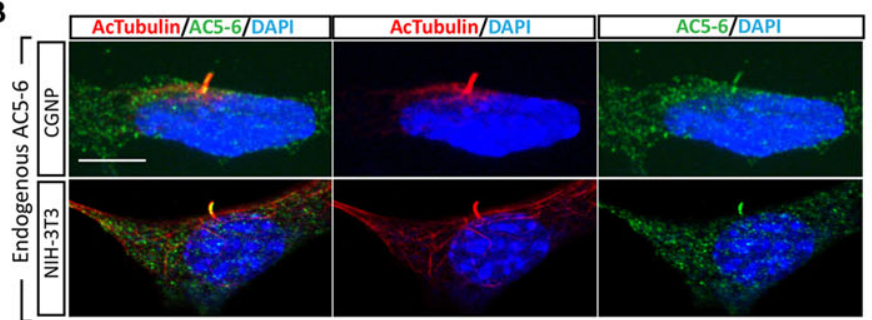

D
C

Rabbit HAPGGSARGSTKKPGGAVTPQQQQQQQRLAAS WR DD-DDDEPPLSGDDPLA 93 Human HAPGGSARGSTKKPGGAVTPQQ---QQRLAS WR DD-DDDDPPLSGDDPLA 91 Mouse HAPGGSTRGSTKRSGGAVTPQQ---QQRLASH WR GGDDDEDPPLSGDDPLA 92 Chicken YPPPPNRSS--------GKKASRMASH WR PEEDYEPQASRSRGESG 66 z.Fish YSARSSC---------KKLIRSNSHWREDDDFERQPGRATTT-- 72
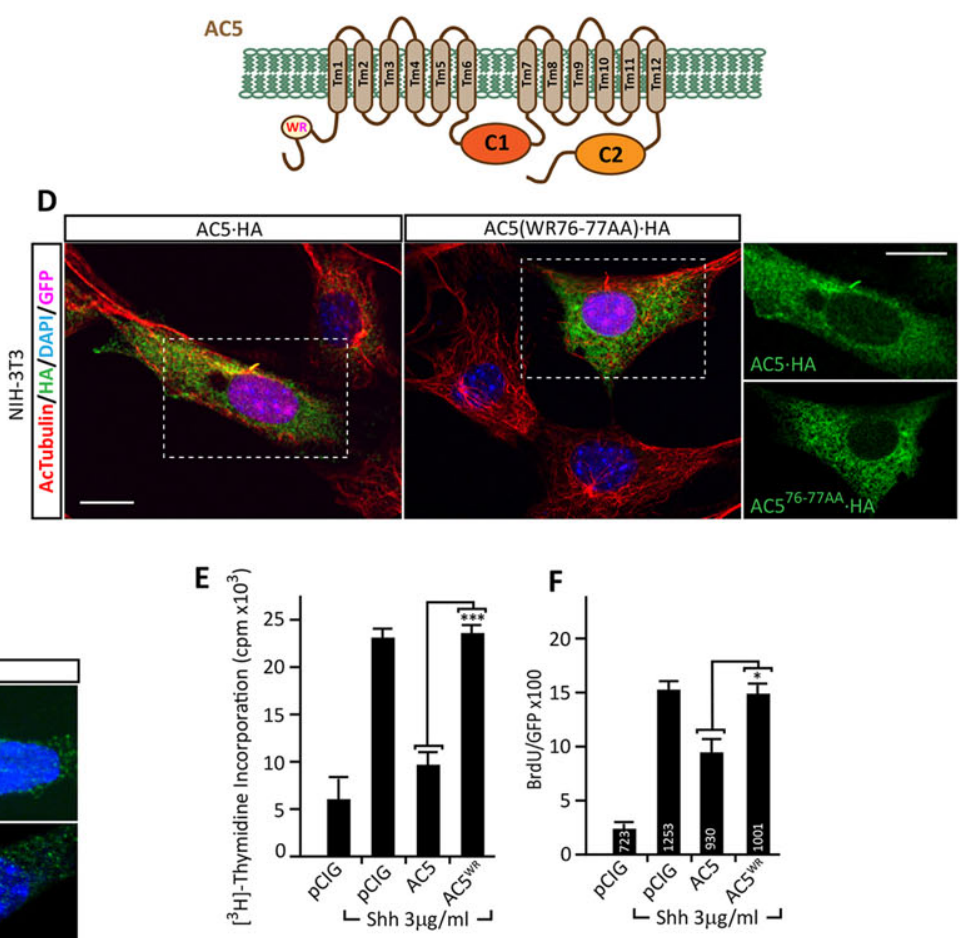

Fig. 2. Adenylyl cyclases require ciliary localization to regulate the Hh pathway. (A) CGNP cultures were transfected for $24 \mathrm{~h}$ with HA-tagged constructs of AC1, AC3, AC5 or AC6 cloned in pCIG, a nuclear-GFP-expressing bicistronic vector. The cells were stained with anti-acetylated-tubulin (AcTubulin, cilium marker, red), anti-HA (green) and anti-GFP (transfection, blue) antibodies. AC3, AC5 and AC6 but not AC1 accumulated in the cilium. (B) Endogenous expression of AC5 and/or AC6 (green) was detected in the cilium of CGNPs and NIH3T3 cells with an antibody that detects both molecules with similar affinity (see supplementary material Fig. S1B). Acetylated Tubulin (red) and DAPI (blue) were used as markers of the cilium and nucleus, respectively. (C) The chart shows inter-species sequence conservation and intramolecular location of a putative AC5 ciliary location motif. The motif was predicted through multiple alignment o

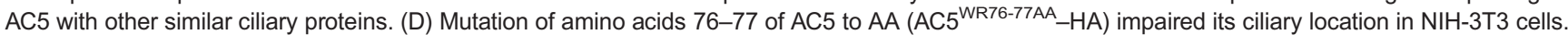

Cultures were stained with anti-AcTubulin (red), anti-HA (green) anti-GFP (purple) antibodies and DAPI (blue). The boxed areas are shown magnified with only the HA staining on the right. (E,F) Shh-induced proliferation of CGNPs was not inhibited by transfection with the AC5 ${ }^{\text {WR76-77AA }}$ mutant as assessed by $\left[{ }^{3} \mathrm{H}\right.$ ] thymidine or BrdU incorporation. All the bar graphs in this figure show the mean \pm s.d. of at least three independent experiments. The total number of cells counted for each data point in panels $\mathrm{F}$ is indicated inside each bar. ${ }^{*} P<0.05,{ }^{* *} P<0.001$ (one-way ANOVA followed by the Tukey's test). Scale bars: $10 \mu \mathrm{m}$. 
an antibody that detects AC5 and AC6 with similar affinity but that does not cross-react with AC3 (supplementary material Fig. S1B), we confirmed that endogenous AC5 and AC6 can be found at the cilia of CGNPs and of NIH-3T cells (Fig. 2B). AC1 was the only adenylyl cyclase isoform that did not diminish Shh-induced proliferation of CGNPs (Fig. 1D,E), therefore a direct relationship between a ciliary localization and the capacity to repress the $\mathrm{Hh}$ pathway was plausible. To study this issue further, we evaluated the possibility that ciliary localization motifs could exist in AC5 by aligning this molecule with other ciliary proteins. Notably, we observed that mutating a WR motif that is also present in Smo (Corbit et al., 2005), involving amino acids 76 and 77 of the rabbit sequence (Fig. 2C), disrupted the distribution of AC5 at the cilium (Fig. 2D). Moreover, although the catalytic activity of the AC5 ${ }^{\text {WR76-77AA }}$ mutant was preserved (supplementary material Fig. S1C,D), it no longer repressed Shh-induced proliferation of CGNPs (Fig. 2E,F).

\section{Expression of AC5 and AC6 represses the Hh pathway in the} developing chicken neural tube

It has been reported that activators of the Hh pathway like GliA or dominant-negative PKA induce overgrowth of neuroepithelial cells and a ventralization of the developing neural tube, whereas repressors like Gli3R reduced cell proliferation and induced dorsalization (Epstein et al., 1996; Stamataki et al., 2005; Cayuso et al., 2006; Dessaud et al., 2008). By using in ovo electroporation and fluorescent cell sorting, we studied the expression of endogenous AC1, AC3, AC5 and AC6 in the neuroblasts of HH12 chicken neural tube at 12 hours post-electroporation (hpe) by q-PCR, and found that, as in CGNPs, AC5 and AC6 were the two isoforms most highly expressed in the neuroblasts of chicken neural tube (Fig. 3A). Thus we studied the effect of AC3, AC5 and AC6 overexpression on the different effects of Shh in the developing chicken neural tube. First, we observed that AC5 or AC6 expression but not that of AC3 significantly reduced cell proliferation in $\mathrm{HH}-12$
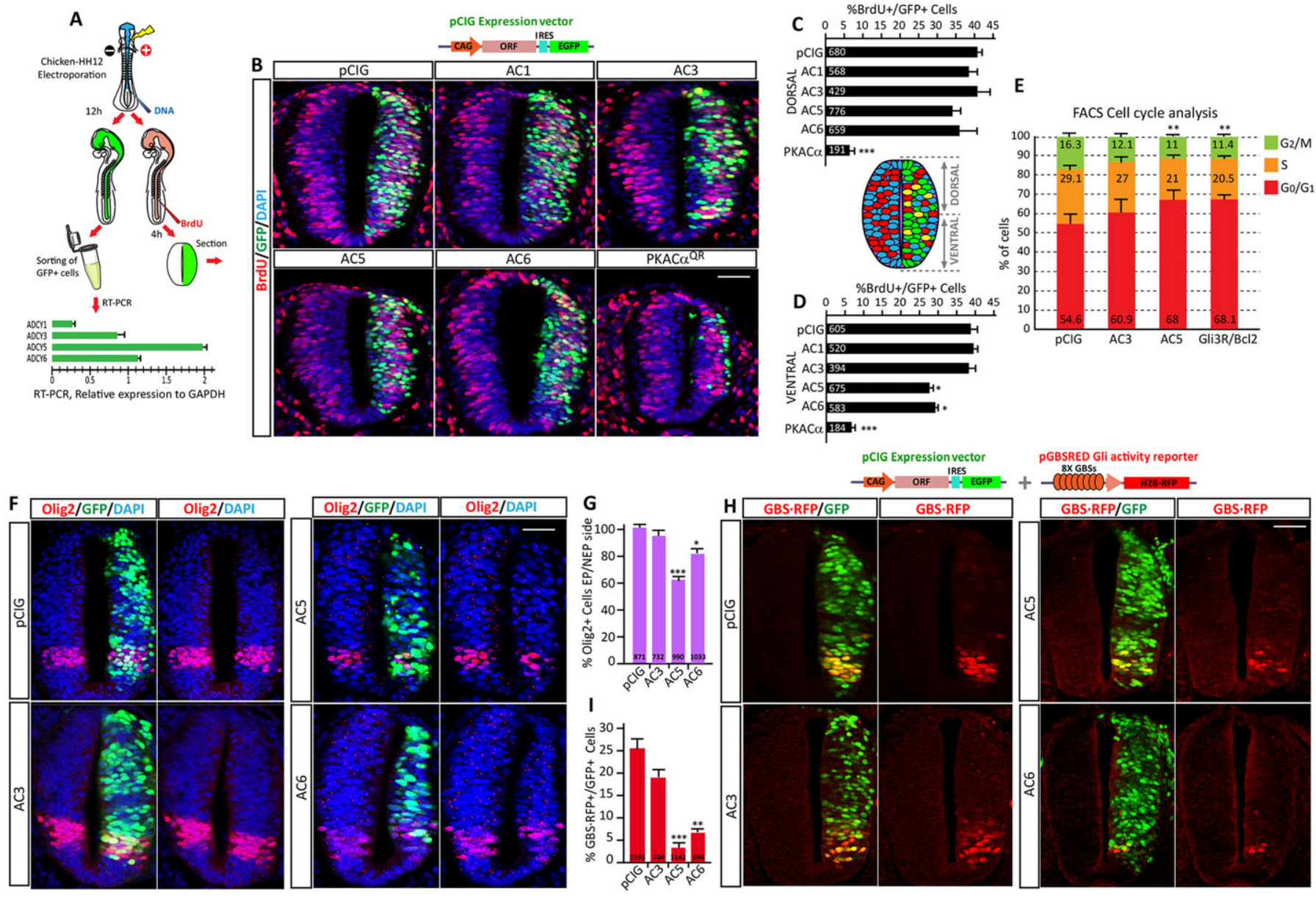

Fig. 3. Expression of $A C 5$ and $A C 6$ represses the Hh pathway in the developing chicken neural tube. (A-D) HH-12 chicken neural tubes were electroporated with different constructs cloned in pCIG (a diagram of the vector is shown above B) and they were left to develop for $16 \mathrm{~h}$. (A) Scheme of the working protocol and expression levels of AC1, AC3, AC5 and AC6 in the neural tubes studied by q-PCR. (B) BrdU $(\sim 1 \mu \mathrm{l}$ at $0.5 \mu \mathrm{g} / \mu \mathrm{l})$ was injected into the neural tube lumen $4 \mathrm{~h}$ prior to fixation and transverse sections of the neural tube were then stained with antibodies against BrdU (proliferation, red) and GFP (transfection, green), and DAPI (nucleus, blue). (C,D) The percentage of BrdU-positive cells among the transfected population was calculated separately for the dorsal and ventral neural tube. PKAC $\alpha^{\mathrm{QR}}$ was used as a positive control. (E) In an experiment similar to that for $A-D$, the cell cycle phase distribution was studied in the population of transfected cells (GFP-positive) by FACS analysis, and the Gli3R and Bcl2 combination was used as a positive control. (F,G) The expression of Olig2 (a marker of motoneuron precursors, red) was studied in $\mathrm{HH}-12$ chicken neural tubes expressing AC3, AC5 or AC6 at 16 hpe (green). The number of Olig2-positive cells in the electroporated side (EP) was compared in each case to the non-electroporated side (NEP). (H,I) HH-12 chicken neural tubes were transfected for $16 \mathrm{~h}$ with AC3, AC5 or AC6 cloned in PCIG (green) together with pGBSRED, a RFP-expressing reporter of Hh pathway activity (red). Diagrams of the vectors are shown above the panels. The percentage of RFP-positive cells among the transfected population is shown in the bar graph. All the bar graphs in this figure show the mean \pm s.d. of at least three independent experiments. The total number of cells counted in each data point in panels $\mathrm{C}, \mathrm{D}, \mathrm{F}$ and $\mathrm{I}$ is indicated inside each bar. ${ }^{*} P<0.05,{ }^{* *} P<0.001$ (one-way ANOVA followed by the Tukey's test). Scale bars: $50 \mu \mathrm{m}$. 
stage chicken embryo neural tubes at 16 hpe with this decrease being most prominent in the ventral part of the tube (Fig. 3B-D). It is worth noting the strong anti-proliferative and pro-differentiative effect developed by the active form of PKA (PKAC $\left.\alpha^{\mathrm{QR}}\right)$, used as a positive control, prevents any proliferative activity between transfection and fixation, enormously reducing the total number of GFP-positive cells (Barzi et al., 2010). In accordance, fluorescence-activated cell sorting (FACS) cell cycle analysis revealed a reduction in the number of cells in the $\mathrm{S}$ and $\mathrm{M}$ phases of the cell cycle in neural tubes transfected with AC5, and it was noteworthy that the distribution of cell cycle phases in neural tubes expressing AC5 was very similar to neural tubes transfected with Gli3R (a Gli3 mutant lacking the transactivation domain) plus Bcl2, this construct combination has been previously reported to block most Shh-dependent signals without inducing apoptosis in the neural tube (Cayuso et al., 2006; Pedersen and Rosenbaum, 2008) (Fig. 3E). Shh also induces the differentiation of motoneurons in the developing neural tube (Marti et al., 1995), stimulating the proliferation and survival of motoneuron precursors (which are Olig2-positive; Cayuso et al., 2006). AC5 or AC6 expression but not that of AC3, significantly reduced the number of Olig2-positive cells at $16 \mathrm{hpe}$ (Fig. 3F,G). Moreover, co-electroporation of adenylyl cyclases with a reporter of $\mathrm{Hh}$ pathway activation $(8 \times$ GBS-RFP), demonstrated that the Hh pathway was activated in fewer of the cells expressing AC5 and AC6 but not in fewer of those expressing AC3 (Fig. 3H,I), a similar result was obtained using the original luciferase expressing vector GBS-LUC (supplementary material Fig. S1E). These results demonstrate that, like in CGNPs, the activity of AC5 and AC6 can suppress the Hh pathway in the neural tube, whereas $\mathrm{AC} 3$ expression has some mild effects on occasions but these effects were never significant in the neural tube.

\section{Knockdown of adenylyl cyclases activates the Hh pathway}

We next wondered whether suppression of adenylyl cyclases was sufficient to stimulate the $\mathrm{Hh}$ pathway and hence, we cloned inhibitory short hairpin RNAs (shRNAs) that target mouse AC3, AC5 or AC6 into the pSHIN vector and probed their effects in CGNP proliferation assays (the sequences targeted and shRNA efficiency are shown in supplementary material Table S1 and Fig. S1F, respectively). Most of the shRNAs designed against AC5 and AC6, but not those against AC3, significantly increased the proliferation of CGNPs both in the presence or absence of low doses of Shh $(0.3 \mu \mathrm{g} / \mathrm{ml}$ : Fig. $4 \mathrm{~A})$, an effect that was not evident at saturating concentrations of Shh (data not shown). We also studied how adenylyl cyclase knockdown affected the Hh pathway during neural tube development using in ovo electroporation of luciferase (Fig. 4B) and RFP (Fig. 4C,D) reporters of Gli activity to test selected chicken shRNAs targeting AC3, AC5 or AC6 (targeted sequences and shRNA efficiency are shown in supplementary material Table S1 and Fig. S1G, respectively). Notably we found that the Hh pathway was activated significantly by AC5 and AC6 knockdown but not by the knockdown of AC3. In addition, the combined suppression of AC5 and AC6 induced further activation of this pathway over and above the knockdown of each enzyme alone. By contrast, combining the knockdown of AC3 and AC5
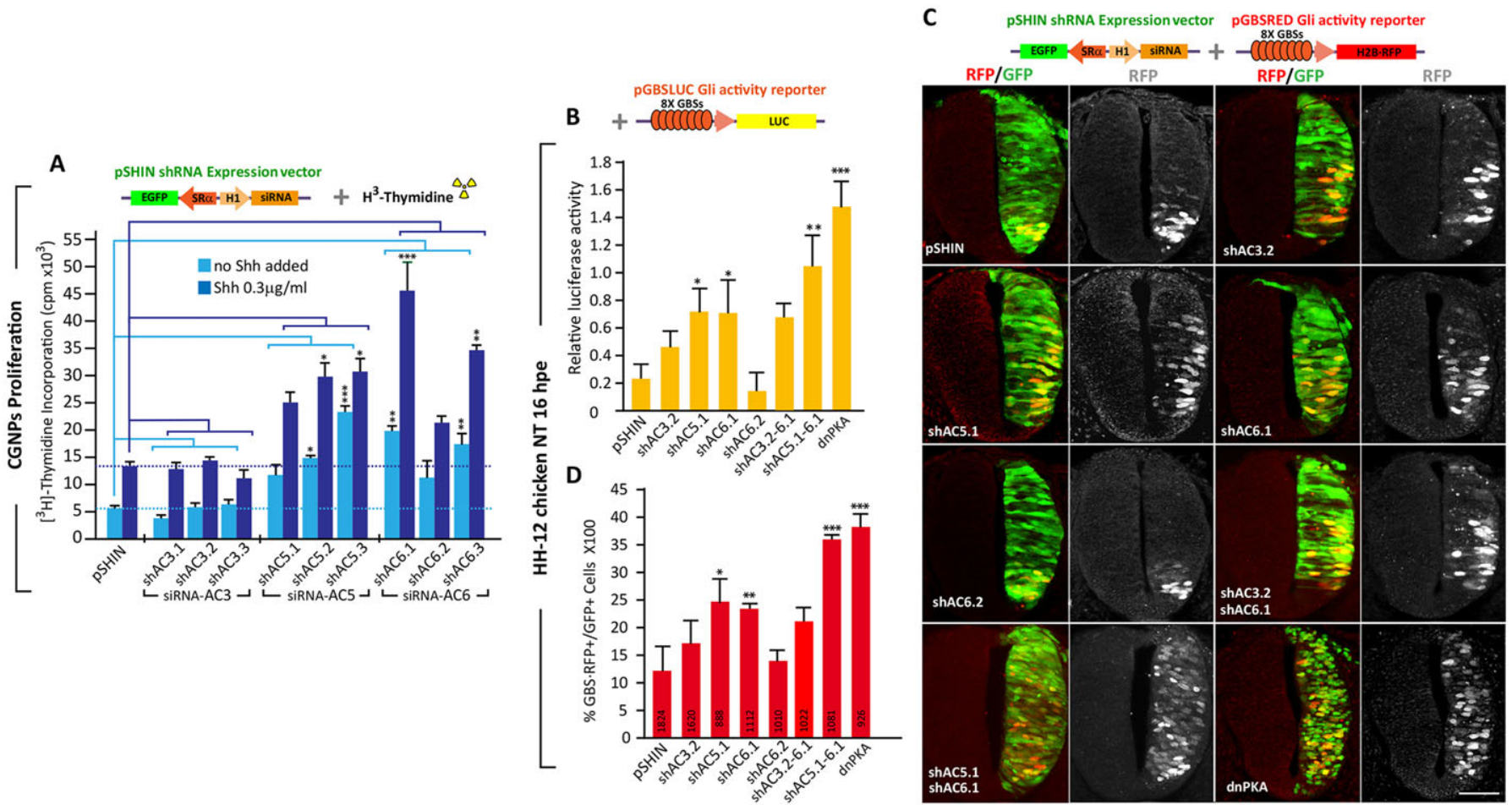

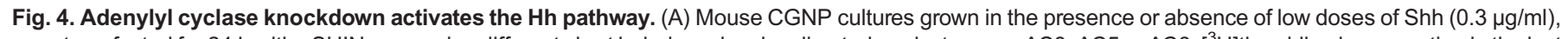
were transfected for $24 \mathrm{~h}$ with pSHIN expressing different short hairpin molecules directed against mouse AC3, AC5 or AC6. [ $\left.{ }^{3} \mathrm{H}\right]$ thymidine incorporation in the last $4 \mathrm{~h}$ of cultures was used to study cell proliferation. (B) $\mathrm{HH}-12$ chicken neural tubes were transfected for $16 \mathrm{~h}$ with pSHIN expressing selected short hairpin molecules directed against AC3, AC5 or AC6, or a combination of these, together with the pGBSLUC luciferase reporter of Hh pathway activity. The efficiency of the different shRNAs is shown in supplementary material Fig. S1F,G. (C,D) As in B but using pGBSRED as the reporter. The dominant-negative (dn)PKA construct was used as a positive control of Hh pathway activation. The percentage of transfected cells that are RFP-positive is shown in the bar graph. Diagrams of the expression vector are shown above the panels. All the bar graphs in this figure show the mean \pm s.d. of at least three independent experiments. The total number of cells counted in each data point in panel $\mathrm{D}$ is indicated inside each bar. ${ }^{*} P<0.05,{ }^{* * *} P<0.001$ (one-way ANOVA followed by the Tukey's test). Scale bar: 50 um. 
activated the Hh pathway to a similar extent as the knockdown of AC5 alone (Fig. 4B-D). As a further control to the empty vector, the effects of an shRNA with very mild activity (shAC6.2) was also evaluated in these experiments. It is worth noting that the stimulatory effect of AC5 and AC6 shRNAs was in all cases reversed by the overexpression AC5 and/or AC6 (data not shown). Thus, these results further support the hypothesis that repression of the Hh pathway in CGNPs and neural tube is mediated by the activity of the ciliary located AC5 and AC6.

\section{The Hh pathway is repressed by $\mathbf{G} \alpha_{s}$ and activated by $\mathbf{G} \alpha_{\mathrm{i}}$ in the developing neural tube}

The proliferation of CGNPs carrying GTPase-deficient mutants of $\mathrm{Go}_{\mathrm{i}} 2$ and $\mathrm{G} \alpha_{\mathrm{i}} 3\left(\mathrm{G \alpha}_{\mathrm{i}} \mathrm{2}^{\mathrm{QL}}\right.$ and $\mathrm{G} \alpha_{\mathrm{i}} 3^{\mathrm{QL}}$, note that $\mathrm{G} \alpha_{\mathrm{i}} 3^{\mathrm{QL}}$ remains active longer) is significantly enhanced when they are grown in the presence of sub-proliferative doses of Shh. Moreover, the proliferation of CGNPs induced by either Shh or SmoM2 is strongly inhibited by the simultaneous knockdown of $\mathrm{G \alpha}_{\mathrm{i}} 2$ and $\mathrm{G}_{\mathrm{i}} 3$ (Barzi et al., 2011). Conversely, $\mathrm{G} \alpha_{\mathrm{s}}$ acts as a tumour suppressor for medulloblastoma and its expression strongly represses CGNP growth (He et al., 2014). Here, we have shown that AC5 and AC6 are the most abundant adenylyl cyclase isoforms in CGNPs, and that in both CGNPs and the chicken neural tube, these adenylyl cyclases exert a relevant effect on the Hh pathway. Additionally, AC5 and AC6 are among the few adenylyl cyclases isoforms that can be simultaneously regulated by $\mathrm{G} \alpha_{\mathrm{s}}$ and $\mathrm{G} \alpha_{\mathrm{i}}$ proteins (Linder, 2006). However, it was not clear whether G $\alpha$ proteins regulate the Hh pathway during neural tube development. We found that like SmoM2, $\mathrm{G \alpha}_{\mathrm{i}} 3^{\mathrm{QL}}$ expression increased the number of Olig2-positive cells, as well as inducing the expression of
Nkx2.2 and Olig2 in cells lying dorsally to their normal domains (Fig. 5A,B,E). Conversely, expression of $\mathrm{G}_{\mathrm{s}}^{\mathrm{RC}}$ (a GTPase-deficient mutant of $G \alpha_{\mathrm{s}}$ ) in $\mathrm{HH}-12$ chicken neural tubes greatly reduced the number of neuroblasts that expressed the $\mathrm{Nkx} 2.2$ and Olig2 ventral markers, with Pax7-positive cells (a dorsal marker) occupying most of the neural tube (Fig. 5A,B,E). Notably, the dorsalization of the neural tube induced by $\mathrm{G}_{\mathrm{s}}^{\mathrm{RC}}$ could be reversed by SmoM2 or $\mathrm{Go}_{\mathrm{i}} 3^{\mathrm{QL}}$ expression, with the maximal ventralization of this tissue observed upon co-expression of SmoM2 and $\mathrm{G \alpha}_{\mathrm{i}} 3^{\mathrm{QL}}$ (Fig. 5C-E).

To obtain more quantitative data on Hh pathway activation in each situation, we used the GBS-Luc reporter and we found that the effects caused by the expression of $\mathrm{G \alpha}_{\mathrm{s}}^{\mathrm{RC}}$ and $\mathrm{G \alpha}_{\mathrm{i}} 3^{\mathrm{QL}}$ on neural tube patterning were correlated with the levels of $\mathrm{Hh}$ pathway activation in the transfected cells (Fig. 5F). Thus, the results presented here demonstrate that, in CGNPs and in the chicken neural tube, the Hh pathway is controlled by ciliary AC5 and AC6, and we present strong evidence suggesting that its activation depends on the associated $\mathrm{G} \alpha_{\mathrm{s}}-\mathrm{G} \alpha_{\mathrm{i}}$ equilibrium.

\section{DISCUSSION}

\section{Cilium adenylyl cyclases regulate the Hh pathway}

In this study, we have investigated the relationship between the PKA and Hh signalling pathways in two tissues in which the latter plays a fundamental role. It has previously been shown that there is an expansion of Shh-dependent ventral cell types in the developing neural tube of PKA-deficient mouse embryos [PKAC $\alpha(\text { Prkaca })^{-/-}$ $P K A C \beta(\text { Prkacb })^{-/+}$or $P K A C \alpha^{-/+} P K A C \beta^{-/-}$; Huang et al., 2002], whereas the neural tube remains open in mice without any catalytic PKA activity $\left(P K A C \alpha^{-/-}, P K A C \beta^{-/-}\right)$and the strong expansion of ventral progenitors is coupled to an absence of dorsal interneuron

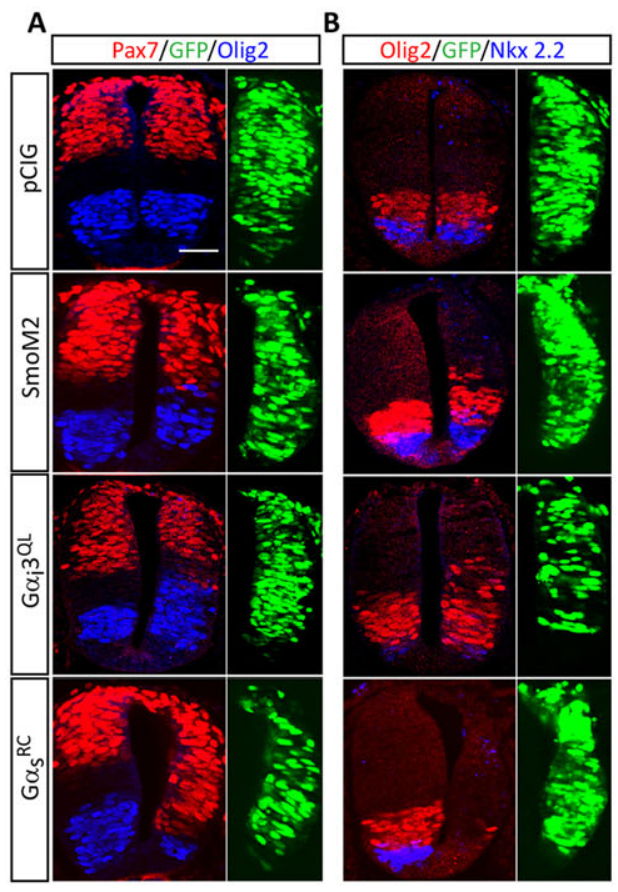

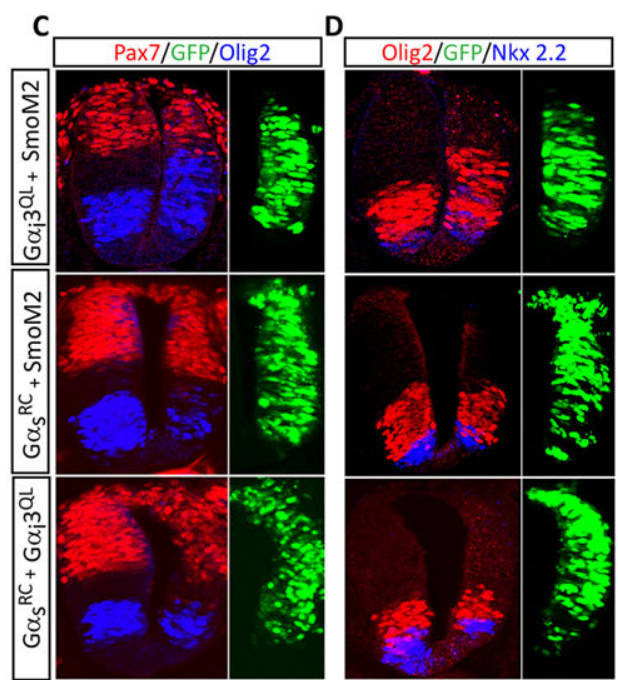
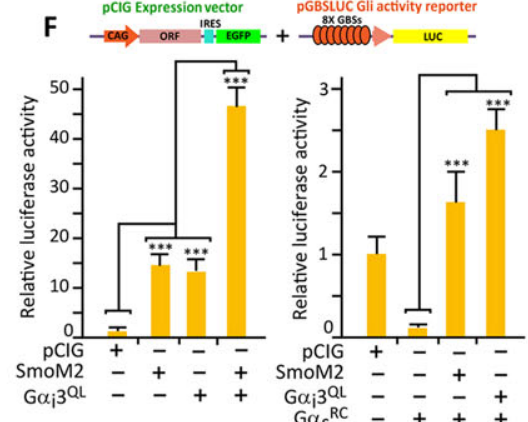

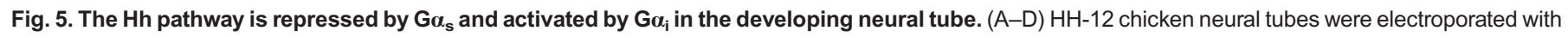
different constructs cloned in pCIG for $24 \mathrm{~h}$. Transverse sections were stained with antibodies against Pax7 (red in A,C), Olig2 (red in B,D; and blue in A,C) and Nkx 2.2 (blue in B,D). GFP expression (green) denotes the transfection intensity and it is shown to the right of each image. (E) Bar graph showing the percentage of positive cells for each maker with respect to the non-electroporated side (left). (F) In an experiment similar to that in E, the activity of the Hh pathway was assessed with the GBS-Luc reporter. Diagrams of the expression vector are shown above the graphs. The bar graphs show the level of Luciferase activity with respect to the control $(\mathrm{pCIG})$ transfected neural tubes. All the bar graphs in this figure show the mean \pm s.d. of at least three independent experiments. The total number of cells counted in each data point in panel $\mathrm{E}$ is indicated inside each bar. ${ }^{*} P<0.05,{ }^{* *} P<0.01,{ }^{* *} P<0.001$ (one-way ANOVA followed by the Tukey's test). Scale bar: $50 \mu \mathrm{m}$. 
progenitors (Tuson et al., 2011). Indeed, this phenotype of PKAnull mice is very similar to that of pthcl-and Sufu-null mice. Thus, the total absence of PKA activity fully emulates maximal activation of the Hh pathway. PKA appears to repress the Hh pathway through two main mechanisms: by preventing Gli2 activation and promoting Gli3 repressor formation. The relevance of each of these mechanisms for $\mathrm{Hh}$ activity varies in the different $\mathrm{Hh}$ target tissues, whereby PKA repression of ventral progenitors in the neural tube seems to be mediated by the inhibition of Gli2 activation (Tuson et al., 2011), yet in tissues, like the limb-bud, Hh repression is mainly achieved by PKA-induced production of the Gli3 repressor (te Welscher et al., 2002).

In recent years, compelling evidence has accumulated showing that the vertebrate Hh pathway fully depends on the integrity of the primary cilium, where activator and repressor forms of Gli transcription factors are generated. Although neither the chronology nor the morphological aspects of the process are as yet clear, a common argument in all the models proposed is the central role played by the pool of PKA located at the cilium base (Tuson et al., 2011; Nozawa et al., 2013; Mukhopadhyay and Rohatgi, 2014). Hence, it would seem that the ciliary components of the Hh pathway exert a tight control over the activation status of this PKA pool. Although the location of PKA at the cilium base is controlled by the A-kinase anchoring proteins (AKAPs; McConnachie et al., 2006; Barzi et al., 2010), its activity is fundamentally controlled by cAMP levels. Although cAMP is fully diffusible, the range at which it effectively regulates PKA activity is largely narrowed down by dilution and phosphodiesterase activity. However, the volume of the cilium is negligible compared to the total cell volume and thus, cAMP can be produced at very high molar concentrations in the cilium where it can control the PKA pool at its base without producing any measurable effects on other PKA pools. Accordingly, we hypothesized that the adenylyl cyclases regulating PKA activity at the cilium base, and therefore controlling the $\mathrm{Hh}$ pathway, should be located at the cilium.

Based on pharmacological data, it has long been assumed that adenylyl cyclases worked as inhibitors of the Hh pathway, although no direct evidence has been available to date. Here, we have shown that among those adenylyl cyclase isoforms expressed at a reasonable level in CGNPs (AC1, AC3, AC5 and AC6), only the isoforms that concentrate in the cilium (AC3, AC5 and AC6) inhibit the proliferation of these cells induced by Shh. Although not demonstrative, these results encouraged us to further confirm our hypothesis and thus, by comparing the sequences of AC5 and AC6 with those of other ciliary proteins, including Smo, we found several putative ciliary location motifs in the AC5 sequence. Notably, we found a WR motive in AC5, that although it was not located close to a transmembrane domain as in Smo or ODR-10, the mutation of which not only inhibited its ciliary localization but also, it abrogated its anti-proliferative effect. Given that the mutant AC5 still induced the phosphorylation of cytoplasmic and nuclear PKA substrates to a similar extent as the wild-type AC5, adenylyl cyclases apparently need to be confined to the cilium to regulate the Hh pathway.

To expand the relevance of our observations, we extended our study to the developing chicken neural tube. Notably, in this structure the expression of AC5 and AC6 significantly reduced different Shh-dependent parameters, such as cell proliferation, Olig2 expression and the activity of the endogenous Hh pathway (as measured with a GBS-RFP reporter). These results demonstrated that ciliary adenylyl cyclases could inhibit the $\mathrm{Hh}$ pathway in different $\mathrm{Hh}$ target tissues. Moreover, knockdown experiments demonstrated that $\mathrm{AC} 5$ and $\mathrm{AC} 6$, but not $\mathrm{AC} 3$, are active repressors of the Hh pathway in both CGNPs and the neural tube, supporting an inhibitory role of ciliary adenylyl cyclases on the Hh pathway. We measured cell proliferation in CGNPs, as we believe it to be the best measure of sustained Hh pathway activation in these cells, ensuring their health and viability. Alternatively, we used reporters of Gli activity to study the effect of adenylyl cyclase suppression in the neural tube. Although the results obtained by these two methods were very similar, the RFP reporter demonstrated that AC5 and AC6 knockdown (like the overexpression of a dominant-negative form of PKA) not only increased the activity of this pathway in the endogenous (ventral) domain but it was also ectopically activated at any dorso-ventral level. These results indicate that AC5 and AC6 actively repress $\mathrm{Hh}$ pathway activation in both CGNPs and the neural tube.

It is worth noting that the AC5 and AC6 double knockdown activated the pathway more strongly than the individual knockdowns alone, almost to the level of the dominant-negative PKA. Hence, it would appear that both AC5 and AC6 contribute to Hh pathway silencing. Although we cannot exclude the contribution of other adenylyl cyclases to this effect, AC5 and AC6 seem to be the principal effectors involved. In this regard, although AC3 overexpression effectively suppressed Shh-induced proliferation in CGNPs, the differences observed when AC3 was overexpressed in the neural tube or when it was knocked-down were never significant. We know that AC3 is endogenously expressed and located in the cilia in both CGNPs and neural tube, yet it is more weakly expressed than AC5 and AC6 in both tissues (data not shown), which could explain the milder effect of AC3 knockdown but not the lack of effect when AC3 is overexpressed in the neural tube. Thus, further studies will be necessary to determine whether AC3 is in fact less effective than AC5 and AC6 in repressing the Hh pathway.

\section{Control of AC5 and AC6 by heterotrimeric G $\alpha$ proteins}

Although adenylyl cyclases have been reported to be modulated by an extensive list of signalling molecules (Linder, 2006), regulation by stimulatory $\left(\mathrm{G} \alpha_{\mathrm{s}}\right)$ and inhibitory $\left(\mathrm{G} \alpha_{\mathrm{i}}\right)$ subunits of heterotrimeric $\mathrm{G}$ proteins occupies a prominent position. Notably, the two adenylyl cyclase isoforms that proved to exert the most relevant effect on the Hh pathway, AC5 and AC6, are also among the few that can be regulated by both stimulatory and inhibitory $G$ proteins. In fact, $\mathrm{G} \alpha_{i}$ inhibits AC5 and AC6 but only when these molecules have been previously activated by $\mathrm{G} \alpha_{\mathrm{s}}$ (Sadana and Dessauer, 2009). Given that the initial studies showing that pertussis toxin can inhibit $\mathrm{G \alpha}_{\mathrm{i}}$ and induce a phenotype resembling Hh deficiency in zebrafish (Hammerschmidt and McMahon, 1998), there is much more evidence that $G \alpha_{i}$ proteins influence the activity of the $\mathrm{Hh}$ pathway (Riobo et al., 2006; Ogden et al., 2008; Barzi et al., 2011; Shen et al., 2013). Nevertheless, it must be recognized that other studies have failed to implicate $\mathrm{G} \alpha_{\mathrm{i}}$ in Hh signalling (Lum et al., 2003; Low et al., 2008). Moreover, Gpr161, a GPCR that signals through $G \alpha_{s}$, has recently been shown to be excluded from the cilium upon activation of the Hh pathway, and there are signs of increased Hh activity in Gpr161-null mice (Mukhopadhyay et al., 2013). In addition, $\mathrm{G} \alpha_{\mathrm{s}}$ suppression has been reported to induce medulloblastoma-like tumours in mice (He et al., 2014). However, it is noteworthy that the phenotype associated with $\mathrm{Hh}$ activation in Gpr161-null mice was very mild compared to that in $\mathrm{G} \alpha_{\mathrm{s}}$ null mice, in which the neural tube undergoes very intense ventralization, similar to that in PKA-null mice (Hwang and Mukhopadhyay, 2015). Thus, as the activity of $\mathrm{G} \alpha_{\mathrm{s}}$ seems to be crucial to prevent the pathway from signalling in the absence of $\mathrm{Hh}$, additional 
mechanisms besides the exclusion of Gpr161 from the cilium seem to be required to counteract $\mathrm{G} \alpha_{\mathrm{s}}$ upon Hh pathway activation.

We have previously reported that $\mathrm{G \alpha}_{\mathrm{i}} 3^{\mathrm{QL}}$ stimulated the proliferation of CGNPs cooperatively with Shh and indeed, combined knockdown of $\mathrm{G}_{\mathrm{i}} 2$ and $\mathrm{G}_{\mathrm{i}} 3$, the two $\mathrm{G} \alpha_{\mathrm{i}}$ subunits located at the cilium, significantly reduce Shh-induced proliferation in these cells (Barzi et al., 2011). Here, we show that in contrast to $\mathrm{G} \alpha_{\mathrm{s}}^{\mathrm{RC}}$, which induces the complete dorsalization of the chicken neural tube, $\mathrm{G} \alpha_{\mathrm{i}} 3^{\mathrm{QL}}$ expression not only increases the number of Olig2-positive cells but that it also extends the Olig2 domain dorsally with a concomitant reduction in the Pax7 territory, similar to the effects of SmoM2. In addition, the extent to which $\mathrm{G \alpha}_{\mathrm{i}} 3^{\mathrm{QL}}$ and SmoM2 reversed the effect of $\mathrm{G}_{\mathrm{s}}^{\mathrm{RC}}$ was very similar. Notably, the effects on neural tube patterning were in all cases correlated to the different extent of $\mathrm{Hh}$ activation evident through the GBS-Luc reporter. It is worth noting that like the results obtained previously in CGNPs (Barzi et al., 2011), we observed significant cooperation between $\mathrm{Go}_{\mathrm{i}} 3^{\mathrm{QL}}$ and SmoM2 in terms of GBS activation in the neural tube, which is consistent with the fact that $\mathrm{G \alpha}_{\mathrm{i}} 3^{\mathrm{QL}}$ must still be engaged by a GPCR to be active. By contrast, no effect on neural tube pattering was associated with $\mathrm{G} \alpha_{\mathrm{i}} 2^{\mathrm{QL}}$ expression (Low et al., 2008), although it must be acknowledged that $\mathrm{G}_{\mathrm{i}}$ plays important roles in cell polarity, and in mitotic spindle orientation and cell division (Siller and Doe, 2009; McCaffrey and Macara, 2011). In fact, there are evident signs of cell depolarization and cell death in $\mathrm{Go}_{\mathrm{i}} 2^{\mathrm{QL}}$ transfected neural tubes (Low et al., 2008). Therefore, the lack of effects on patterning reported upon $\mathrm{G}_{\mathrm{i}} 2^{\mathrm{QL}}$ expression should be interpreted with caution. So far we are not able to confirm whether $\mathrm{G} \alpha_{\mathrm{i}}$ is part of the Hh pathway in the neural tube, as observed in CGNPs, nor if it is directly activated by Smo or by another GPCR activated by it. However, our results clearly support the plausibility of considering $\mathrm{G} \alpha_{i}$ to be being part of the Hh pathway in the neural tube.

\section{MATERIALS AND METHODS}

\section{Antibodies and chemicals}

The different antibodies used in this study were: mouse monoclonal antibodies against Gli1 (Cell Signaling, MAB2643), Nkx2.2 (DSHB, 74.5A5), Pax7 (DSHB), $\alpha$-tubulin (Sigma, T6199), acetylated tubulin (Sigma, 6-11B-1), FoxA2 (DSHB, 4c7); a rat monoclonal antibody against BrdU (AbD-Serotec, BU1/75); rabbit polyclonal antisera against phosphorylated (at serine and threonine residues) PKA substrates (PKAPS; Cell Signaling 9621), Olig2 (Millipore, AB9610), AC3 (Santa Cruz, Sc588), both AC5 and AC6 (Santa Cruz SC-590), and GFP, RFP, FLAG and HA (produced in-house).

Alexa-Fluor- and peroxidase-labelled secondary antibodies were obtained from Molecular Probes and Pierce, respectively, whereas BrdU was purchased from Sigma and Rhodamine-Phalloidin from Invitrogen.

\section{DNA constructs}

The bovine $A D C Y 1$ cDNA was obtained from Xavier Nicol (Institute de la vision, France), rat $A D C Y 3$ from Naoyuki Taniguchi (Department of Biochemistry, Osaka University, Japan), rabbit ADCY5 from Joachim Schultz (Pharmazeutisches Institut, University of Tübingen, Germany), human ADCY6 from Rennolds Ostrom (Department of Pharmacology, University of Tennessee, USA), human SmoM2 from Frederic J. Sauvage (Department of Molecular Oncology at Genentech, Inc., USA), and rat Gnas and Gnas ${ }^{\mathrm{R} 201 \mathrm{C}}$ from Natalia Riobo (Department of Biochemistry, Thomas Jefferson University, USA). Gli3R is a deleted form of human Gli3 encoding amino acids 1-768 (Persson et al., 2002). The luciferase reporter was provided by Yongbin Chen (Department of Developmental Biology, University of Texas, USA) and the RFP Gli-binding site (GBS) reporter was produced in our laboratory. All the constructs were cloned into pCIG, an EGFP-expressing bicistronic vector. Site-directed mutagenesis, and the addition of HA and FLAG tags was achieved by PCR. By contrast, inhibitory short hairpin RNAs
(shRNA) were cloned into pSHIN (a GFP-expressing evolution of pSUPER). The shRNA constructs where electroporated in CGNPs with the Microporator MP-100 (see below) using $10 \mu \mathrm{g}$ of DNA per $3 \times 10^{6}$ cells in $100 \mu \mathrm{l}$ of transfection solution. In the neural tube, the shRNA constructs were diluted at $1 \mu \mathrm{g} / \mu \mathrm{l}$ and $\sim 1 \mu \mathrm{l}$ was used for each chicken neural tube electroporation. The list of sequences targeted by the shRNAs and all the primers used for q-PCR are shown in the supplementary material Table S1.

\section{CGNP cultures and transfection}

Cerebellar cultures were prepared using a modification of the Papain method, as described previously (Rios et al., 2004). For transient transfections, freshly isolated cells were electroporated in suspension and plated on dishes coated in poly-L-Lysine plus laminin in Neurobasal plus B-27 media (Invitrogen) supplemented with $\mathrm{KCl}(25 \mathrm{mM})$, glutamine $(1 \mathrm{mM})$ and Shh $(3 \mu \mathrm{g} / \mathrm{ml})$. After $24 \mathrm{~h}$, the medium was replaced by fresh medium containing the corresponding treatments, taking this moment as time 0. Electroporation was performed using the Microporator MP-100 (Digital Bio, Seoul, Korea) according to the manufacturer's instructions, with a single pulse of $1700 \mathrm{~V}$ for $20 \mathrm{~ms}$. The NIH-3T3 and HEK293 cell lines were grown in Dulbecco's modified Eagle's medium (DMEM) containing $10 \%$ fetal bovine serum (FBS)

\section{Chick embryo in ovo electroporation}

White-Leghorn chick embryos were staged according to Hamburger and Hamilton (1992), and electroporated with column purified plasmid DNA $(1-2 \mu \mathrm{g} / \mathrm{ml})$ in water containing Fast Green $(50 \mathrm{ng} / \mathrm{ml})$. Transfected embryos were allowed to develop to the stages specified before they were processed as indicated. All animal experiments were performed according to approved guidelines.

\section{In situ hybridization}

The cerebellum from P7 mice was fixed overnight in 4\% paraformaldehyde and washed three times for $10 \mathrm{~min}$ with PBT $(1 \times$ DEPC-PBS, $0.1 \%$ Triton $\mathrm{X}-100$ ). The tissue was embedded in $10 \%$ sacarose and $5 \%$ agarose, and vibratome sections $(50 \mu \mathrm{m}$, Leica) were obtained and dehydrated with increasing concentrations of methanol in PBT $(25 \%, 50 \%, 75 \%$ and $100 \%)$. Hybridization was performed at $70^{\circ} \mathrm{C}$ using standard procedures and the probe distribution was detected with alkaline-phosphatase-coupled antidigoxigenin Fab fragments (Boehringer Mannheim). RNA probes were produced from the corresponding cDNA sequences cloned in Bluescript-IIKS with the T7 polymerase.

\section{CGNP proliferation assays - $\left[{ }^{3} \mathrm{H}\right]$ thymidine or BrdU incorporation}

Electroporated cells were plated for $24 \mathrm{~h}$ and then given a 4-h pulse of either $\left[{ }^{3} \mathrm{H}\right]$ thymidine $\left[1 \mu \mathrm{Ci}\left(3.7 \times 10^{4} \mathrm{~Bq}\right)\right.$ each well, from Amersham, Buckinghamshire, UK] or BrdU $(5 \mu \mathrm{g} / \mathrm{ml})$. The cells that received $\left[{ }^{3} \mathrm{H}\right]$ thymidine were then lysed in SDS $(0.04 \%)$ and proliferation was measured in a Wallac scintillation counter (Wallac-Perkin Elmer, Quebec, Canada). The BrdU-labelled cells were fixed and immunostained with an antibody against BrdU, and cell proliferation was calculated as the proportion of the transfected population (GFP expressing cells) that incorporated BrdU.

\section{FACS}

HH-12 chicken embryos were electroporated with the different constructs cloned in pCIG and at $24 \mathrm{hpe}$, a single-cell suspension was obtained by digestion for 10-15 min with Trypsin-EDTA (Sigma). At least three independent experiments were analysed by FACS (six embryos in each experimental condition). Hoechst and GFP fluorescence was determined by flow cytometry in a MoFlo cytometer (DakoCytomation, Fort Collins, CO), and the cellular DNA content was analysed (ploidy analysis) in single fluorescence histograms using Multicycle software (Phoenix Flow Systems, San Diego, CA).

\section{Immunohistochemistry and BrdU incorporation in chicken} embryos

Embryos were fixed in $4 \%$ paraformaldehyde for $2 \mathrm{~h}$ at $4{ }^{\circ} \mathrm{C}$ and immunostaining was performed on vibratome $(40 \mu \mathrm{m})$ sections following 
standard procedures. BrdU $(\sim 1 \mu \mathrm{l}$ at $0.5 \mu \mathrm{g} / \mu \mathrm{l})$ was injected into the neural tube lumen $4 \mathrm{~h}$ prior to fixation and transverse sections of the neural tube were then stained with antibodies against BrdU (proliferation, red) and GFP (transfection, green), and DAPI (nucleus, blue). The total number of GFP-positive (transfected cells) and the number of GFP and BrdU double-labelled cells (transfected that incorporated BrdU) were counted for each slice and the percentage BrdU positive among the transfected population calculated. The percentage was calculated separately for the dorsal and ventral neural tube. To detect BrdU, sections were incubated in $2 \mathrm{~N} \mathrm{HCl}$ for $30 \mathrm{~min}$ and then rinsed with $0.1 \mathrm{M}$ $\mathrm{Na}_{2} \mathrm{~B}_{4} \mathrm{O}_{7}$ ( $\mathrm{pH}$ 8.5). After washing in PBS with $0.1 \%$ Triton X-100, the sections were incubated with the appropriate primary antibodies, the binding of which was detected with Alexa-Fluor-conjugated secondary antibodies. After staining, the sections were mounted and examined on a Leica SP5 confocal microscope.

\section{Immunoblotting}

For immunobloting, transfected cells were dissolved in 1× SDS Laemmli sample buffer (five embryos per condition) and after sonication, the insoluble material was removed by centrifugation. Samples were resolved by $8 \%$ SDS-PAGE, transferred to nitrocellulose membranes, blocked with $8 \%$ non-fat dried milk powder in TTBS $(150 \mathrm{mM} \mathrm{NaCl}, 0.05 \%$ Tween-20 and $20 \mathrm{mM}$ Tris-HCl $\mathrm{pH}$ 7.4) and the membranes were probed with the primary antibodies. Antibody binding to the blots was detected using protein A/G-coupled peroxidase that was visualized with the ECL system and captured with a Versadoc Imaging System (Bio-Rad), or with AlexaFluor-labelled secondary antibodies that were visualized with the Odyssey Infrared Imaging System (LI-COR). The expression was quantified with Quantity One software (Bio-Rad) and the molecular masses of the proteins estimated using Bio-Rad Precision Molecular Weight Markers.

\section{Luciferase reporter assay of Gli activity}

CGNP cultures or HH-12 chicken embryos were electroporated with the DNAs indicated together with a GBS-Luciferase reporter construct containing eight synthetic Gli-binding sites (GBS-Luc) and a CMV driven Renilla construct (Promega) for electroporation normalization. Total CGNP cultures or GFP-positive neural tubes were dissected out at 48 hpe and homogenized in Passive Lysis Buffer (Promega). Firefly and Renilla luciferase activity was measured with the Dual Luciferase Reporter Assay System (Promega).

\section{Quantitative fluorescence image analysis}

PKA activity was evaluated using an antibody raised against phosphorylated PKA targets, as reported previously (Barzi et al., 2010; He et al., 2014). The relative amount of phosphorylated (at serine and threonine residues) PKA substrates (PKA-PS) was compared between HEK-293 cells transfected with either wild-type AC5 or the mutant AC5 ${ }^{\text {WR76-77AA }}$. Confocal images were acquired with a laser-scanning confocal microscope (Sp5, Leica). Maximum projections of three consecutive confocal sections $(2 \mu \mathrm{m}$ total $\mathrm{Z})$ were quantified with ImageJ software (National Institutes of Health), converting all images into 32 bits and subtracting the background using the original thresholds set with the background as 'NaN' ('not a number'). Finally, the mean grey value was quantified from an area containing the entire cell. The ratio between GFP (transfection level) and PKA-PS was calculated for each cell.

\section{Reverse transcriptase real-time PCR}

Total RNA extracts were obtained following the TRIzol protocol (Invitrogen). Reverse transcription and real-time PCR (q-PCR) were performed according to the manufacturer's instructions (Roche) using a lightcycler (LC 480; Roche).

\section{Acknowledgements}

Monoclonal antibodies were obtained from the Developmental Studies Hybridoma Bank, developed under the auspices of the NICHD and maintained by The University of lowa (Department of Biological Sciences, lowa City, IA 52242).

\section{Competing interests}

The authors declare no competing or financial interests.

\section{Author contributions}

S.P. conceived the study, L.V. designed performed and analysed most of the experiments, A.H. did neural tube patterning experiments; B.T. performed BrdU incorporation and short hairpin cloning duties. A.M. provided technical assistance, S.P. wrote the paper.

\section{Funding}

This work was supported by Spanish and Catalan ministries of Science [grant numbers BFU2008-02424/BFI, BFU2011-24099 and SGR200821A333 to S.P.]

\section{Supplementary material}

Supplementary material available online at

http://jcs.biologists.org/lookup/suppl/doi:10.1242/jcs.172635/-/DC1

\section{References}

Barzi, M., Berenguer, J., Menendez, A., Alvarez-Rodriguez, R. and Pons, S. (2010). Sonic-hedgehog-mediated proliferation requires the localization of PKA to the cilium base. J. Cell Sci. 123, 62-69.

Barzi, M., Kostrz, D., Menendez, A. and Pons, S. (2011). Sonic Hedgehoginduced proliferation requires specific Galpha inhibitory proteins. J. Biol. Chem. 286, 8067-8074.

Briscoe, J. and Thérond, P. P. (2013). The mechanisms of Hedgehog signalling and its roles in development and disease. Nat. Rev. Mol. Cell Biol. 14, 416-429.

Cayuso, J., Ulloa, F., Cox, B., Briscoe, J. and Marti, E. (2006). The Sonic hedgehog pathway independently controls the patterning, proliferation and survival of neuroepithelial cells by regulating Gli activity. Development 133 , 517-528.

Corbit, K. C., Aanstad, P., Singla, V., Norman, A. R., Stainier, D. Y. R. and Reiter, J. F. (2005). Vertebrate Smoothened functions at the primary cilium. Nature 437, 1018-1021.

Dahmane, N. and Ruiz i Altaba, A. (1999). Sonic hedgehog regulates the growth and patterning of the cerebellum. Development 126, 3089-3100.

Dessaud, E., McMahon, A. P. and Briscoe, J. (2008). Pattern formation in the vertebrate neural tube: a sonic hedgehog morphogen-regulated transcriptional network. Development 135, 2489-2503.

Epstein, D. J., Marti, E., Scott, M. P. and McMahon, A. P. (1996). Antagonizing cAMP-dependent protein kinase $A$ in the dorsal CNS activates a conserved Sonic hedgehog signaling pathway. Development 122, 2885-2894.

Hamburger, V. and Hamilton, H. L. (1992). A series of normal stages in the development of the chick embryo. 1951. Dev. Dyn. 195, 231-272.

Hammerschmidt, M. and McMahon, A. P. (1998). The effect of pertussis toxin on zebrafish development: a possible role for inhibitory G-proteins in hedgehog signaling. Dev. Biol. 194, 166-171.

He, X., Zhang, L., Chen, Y., Remke, M., Shih, D., Lu, F., Wang, H., Deng, Y., Yu, Y., Xia, Y. et al. (2014). The $G$ protein alpha subunit $\mathrm{G} \alpha \mathrm{s}$ is a tumor suppressor in Sonic hedgehog-driven medulloblastoma. Nat. Med. 20, 1035-1042.

Huang, Y., Roelink, H. and McKnight, G. S. (2002). Protein kinase A deficiency causes axially localized neural tube defects in mice. J. Biol. Chem. 277 19889-19896.

Hwang, S.-H. and Mukhopadhyay, S. (2015). G-protein-coupled receptors and localized signaling in the primary cilium during ventral neural tube patterning. Birth Defects Res. A Clin. Mol. Teratol. 103, 12-19.

Le Dréau, G. and Martí, E. (2012). Dorsal-ventral patterning of the neural tube: a tale of three signals. Dev. Neurobiol. 72, 1471-1481.

Linder, J. U. (2006). Class III adenylyl cyclases: molecular mechanisms of catalysis and regulation. Cell. Mol. Life Sci. 63, 1736-1751.

Low, W.-C., Wang, C., Pan, Y., Huang, X.-Y., Chen, J. K. and Wang, B. (2008). The decoupling of Smoothened from Galphai proteins has little effect on Gli3 protein processing and Hedgehog-regulated chick neural tube patterning. Dev. Biol. 321 , $188-196$

Lum, L., Yao, S., Mozer, B., Rovescalli, A., Von Kessler, D., Nirenberg, M. and Beachy, P. A. (2003). Identification of Hedgehog pathway components by RNAi in Drosophila cultured cells. Science 299, 2039-2045.

Marti, E., Bumcrot, D. A., Takada, R. and McMahon, A. P. (1995). Requirement of $19 \mathrm{~K}$ form of Sonic hedgehog for induction of distinct ventral cell types in CNS explants. Nature $\mathbf{3 7 5}, 322-325$.

McCaffrey, L. M. and Macara, I. G. (2011). Epithelial organization, cell polarity and tumorigenesis. Trends Cell Biol. 21, 727-735.

McConnachie, G., Langeberg, L. K. and Scott, J. D. (2006). AKAP signaling complexes: getting to the heart of the matter. Trends Mol. Med. 12, 317-323.

Mukhopadhyay, S. and Rohatgi, R. (2014). G-protein-coupled receptors, Hedgehog signaling and primary cilia. Semin. Cell Dev. Biol. 33, 63-72.

Mukhopadhyay, S., Wen, X., Ratti, N., Loktev, A., Rangell, L., Scales, S. J. and Jackson, P. K. (2013). The ciliary G-protein-coupled receptor Gpr161 negatively regulates the Sonic hedgehog pathway via cAMP signaling. Cell 152, 210-223.

Nozawa, Y. I., Lin, C. and Chuang, P.-T. (2013). Hedgehog signaling from the primary cilium to the nucleus: an emerging picture of ciliary localization, trafficking and transduction. Curr. Opin. Genet. Dev. 23, 429-437. 
Ogden, S. K., Fei, D. L., Schilling, N. S., Ahmed, Y. F., Hwa, J. and Robbins, D. J. (2008). G protein Galphai functions immediately downstream of Smoothened in Hedgehog signalling. Nature 456, 967-970.

Pan, Y., Bai, C. B., Joyner, A. L. and Wang, B. (2006). Sonic hedgehog signaling regulates Gli2 transcriptional activity by suppressing its processing and degradation. Mol. Cell. Biol. 26, 3365-3377.

Pedersen, L. B. and Rosenbaum, J. L. (2008). Intraflagellar transport (IFT) role in ciliary assembly, resorption and signalling. Curr. Top. Dev. Biol. 85, 23-61.

Persson, M., Stamataki, D., te Welscher, P., Andersson, E., Böse, J., Rüther, U. Ericson, J. and Briscoe, J. (2002). Dorsal-ventral patterning of the spinal cord requires Gli3 transcriptional repressor activity. Genes Dev. 16, 2865-2878.

Riobo, N. A., Saucy, B., DiLizio, C. and Manning, D. R. (2006). Activation of heterotrimeric $G$ proteins by Smoothened. Proc. Natl. Acad. Sci. USA 103, 12607-12612.

Rios, I., Alvarez-Rodríguez, R., Marti, E. and Pons, S. (2004). Bmp2 antagonizes sonic hedgehog-mediated proliferation of cerebellar granule neurones through Smad5 signalling. Development 131, 3159-3168.

Rohatgi, R., Milenkovic, L. and Scott, M. P. (2007). Patched1 regulates hedgehog signaling at the primary cilium. Science $317,372-376$

Sadana, R. and Dessauer, C. W. (2009). Physiological roles for G protein-regulated adenylyl cyclase isoforms: insights from knockout and overexpression studies. Neurosignals 17, 5-22.
Shen, F., Cheng, L., Douglas, A. E., Riobo, N. A. and Manning, D. R. (2013) Smoothened is a fully competent activator of the heterotrimeric $\mathrm{G}$ protein $\mathrm{G}(\mathrm{i})$. Mol. Pharmacol. 83, 691-697.

Siller, K. H. and Doe, C. Q. (2009). Spindle orientation during asymmetric cell division. Nat. Cell Biol. 11, 365-374.

Stamataki, D., Ulloa, F., Tsoni, S. V., Mynett, A. and Briscoe, J. (2005). A gradient of Gli activity mediates graded Sonic Hedgehog signaling in the neural tube. Genes Dev. 19, 626-641.

te Welscher, P., Zuniga, A., Kuijper, S., Drenth, T., Goedemans, H. J., Meijlink, F. and Zeller, R. (2002). Progression of vertebrate limb development through SHHmediated counteraction of GLI3. Science 298, 827-830.

Tempe, D., Casas, M., Karaz, S., Blanchet-Tournier, M.-F. and Concordet, J.-P. (2006). Multisite protein kinase A and glycogen synthase kinase 3beta phosphorylation leads to Gli3 ubiquitination by SCFbetaTrCP. Mol. Cell. Biol. 26, 4316-4326.

Tuson, M., He, M. and Anderson, K. V. (2011). Protein kinase A acts at the basal body of the primary cilium to prevent Gli2 activation and ventralization of the mouse neural tube. Development 138, 4921-4930.

Wang, B., Fallon, J. F. and Beachy, P. A. (2000). Hedgehog-regulated processing of $\mathrm{Gli3}$ produces an anterior/posterior repressor gradient in the developing vertebrate limb. Cell 100, 423-434.

Wechsler-Reya, R. J. and Scott, M. P. (1999). Control of neuronal precursor proliferation in the cerebellum by Sonic Hedgehog. Neuron 22, 103-114. 